\title{
CRYSTALLINE VARIATIONAL PROBLEMS
}

\author{
BY JEAN E. TAYLOR
}

Surface tension is commonly thought of as a fluid phenomenon; the mere mention of the term brings to mind bugs skimming over water, liquids rising or falling in capillary tubes-and soap films and soap bubbles. But there is in fact a notion of surface tension (which is surface energy per unit surface area) for the interface between any two substances, or even between one substance and a vacuum. This surface energy arises from the fact that atoms (or molecules, or ions) of a given substance have a different environment at the interface between that substance and another than those in the bulk of the substance. (Sometimes even the composition of the surface is different from the bulk; this occurs for instance in soapy water having an interface with air.)

In this article we will deal with "surface tension functions" which are an outgrowth of the surface tensions of solids having their atoms arranged in some regular way. If one fixes the orientation of the lattice in $R^{3}$, then the environment of an atom on a planar interface between something else and such a regular structure can be different for different plane directions. Thus the surface tension between one substance and another can be a function

$$
F: G_{0}(3,2) \rightarrow R^{+}
$$

where $G_{0}(3,2)$ is the Grassmannian of oriented 2-planes through the origin in $R^{3}$ and $R^{+}$is the set of positive real numbers. For the interface between, say, soapy water and air, the function $F$ would be identically a constant; but for that between, say, a single crystal of ordinary salt $(\mathrm{NaCl})$ and air, $F$ would depend quite strongly on direction. If one wishes to allow the regular structure to vary over relatively long distances, one can even obtain a function

$$
F: R^{3} \times G_{0}(3,2) \rightarrow R^{+} .
$$

(This notion of surface tension for solid bodies is not at all esoteric, by the way: it is commonly used by metallurgists and others who deal with the surface properties of materials.)

Article based on an invited hour address to the Eastern Sectional Meeting of the American Mathematical Society in Wellesley, Massachusetts, October 22, 1977; received by the editors October 5, 1977.

AMS (MOS) subject classifications (1970). Primary 49F22, 49F20, 53C65, 49D99, 82A60, 82A65, 52A05, 52A40, 28A75, 49F10.

Key words and phrases. Calculus of variations, geometric measure theory, crystals, surface tension, approximation of minimal surfaces, prescribed boundary problem.

'This work was partially supported by NSF Grant MCS76-06424 and by a Sloan Fellowship. 
Functions from $G_{0}(3,2)$ to $R^{+}$are already well known in the calculus of variations as treated in the context of geometric measure theory. They are called 2-dimensional constant coefficient oriented integrands on $R^{3}$, since they can be integrated over any surface $S$ which has an oriented tangent plane $\operatorname{Tan}(S, x)$ at at least almost every point: the integral of $F$ over $S$ is then $\int_{S} F(\operatorname{Tan}(S, x)) d \mathcal{H}^{2} x$. (Here $\mathcal{H}^{2}$ is Hausdorff 2-dimensional area, which agrees with any other reasonable notion of area on smooth 2-dimensional submanifolds of $R^{3}$ and additionally gives a precise meaning to the area of surfaces with singularities.) The typical problem is to minimize the integral of an integrand, subject to various possible side conditions such as a surface having a given boundary (in any one of a variety of senses) and/or enclosing one or more given volumes. For integrands coming from surface tension, this amounts to finding the surface of least energy satisfying the side conditions (we are, of course, neglecting here a large number of other possible contributions to energy, such as energy from curvature-including edges and corners-but the above is still usually a reasonable model). Since physical systems are in equilibrium if and only if they are at a (local) minimum of total energy, it is of great importance to know such things as the existence, uniqueness, regularity, singularity structure, and construction of shapes minimizing surface energy.

Mathematically, these questions have been addressed in the past primarily for the area integrand $F \equiv 1$ (minimizing the integral of this integrand produces minimal surfaces), and more generally for smooth elliptic integrands [A1]. But integrands which are surface tension functions of solids with regular lattice structures are not elliptic, as will be indicated in $\$ \S 2$ and 3.

A class of nonelliptic integrands which include such surface tension functions is isolated below and called crystalline. My object is to consider all the above problems-existence, uniqueness, singularity structure, and construction-for surfaces having a prescribed boundary and minimizing the integral of a crystalline integrand on $R^{3}$. It will be illustrated how this knowledge can be used to give more information on surfaces minimizing the integrals of any integrand on $R^{3}$. Many of the results of this article already extend to higher dimensions; extension of the others is an active area of research.

This article is in seven sections. The first gives a classical construction, the Wulff construction, for a given integrand $F$ and a proof that the result of the construction is the shape which has uniquely the least surface integral for the volume it contains. This shape is called the crystal of the integrand $F$, and $F$ 's whose crystals are polyhedral are called crystalline. The second section gives examples of integrands and their crystals. The third details the relationship between crystalline integrands and other integrands, and between general crystalline integrands and convex crystalline integrands. The fourth section produces the most fundamental F-minimal surfaces for the prescribed boundary problem.

In the fifth section, a volume-maximizing criterion is given for selecting an F-minimal surface in case there is more than one such solution for a given boundary; in the sixth section, such a volume-maximizing F-minimal surface for a crystalline $F$ is shown to have its tangent planes restricted to a specific 
finite set provided a condition is imposed on the boundary (boundaries satisfying this condition are, however, $C^{0}$-dense in the space of all boundaries). Finally, in $\$ 7$ a procedure for the explicit construction of F-minimal surfaces satisfying an additional boundary condition is outlined (without proof). By the results of \$2, this procedure produces explicit approximations to a surface minimizing the integral of any given integrand.

Before we begin, however, a little terminology is in order. (For the terminology of geometric measure theory in general, see [F1] or [F2].) The class of surfaces to consider in minimizing surface energy should involve oriented surfaces of finite area forming at least part of the boundary of a region and having a well-defined oriented unit normal, at least $\mathcal{H}^{2}$ almost everywhere. Such surfaces, if they also have nice boundaries, are represented naturally by elements of the class $I_{2}\left(R^{3}\right)$ of integral currents of dimension 2 on $R^{3}$. For the purely mathematical questions, also, integral currents are a natural domain of the problem. A 2-dimensional integral current $S$ considered in this article has in particular the following ingredients: (1) a measure $\|S\|$ on $R^{3}$, which is simply a positive-integer-valued function $m$ (the multiplicity) times $S C^{2}$ restricted to a set, spt $S$, which can be arbitrarily closely approximated (in $\mathcal{F C}^{2}$ measure) by a 2-dimensional $C^{1}$ manifold, (2) a $G_{0}(3,2)$-valued function $\vec{S}$ defined for $\|S\|$ almost all $x ; \vec{S}(x)$ is just the oriented tangent plane to that underlying set at $x$, and (3) an oriented boundary, $\partial S$, which has properties like (1) and (2) but with 1 replacing 2 throughout. Thus the integral $\mathrm{F}(S)$ of $F$ over the integral current $S$ is

$$
\mathbf{F}(S)=\int F(\vec{S}(x)) d\|S\| x .
$$

The mass of $S, M(S)$, is defined to be $\|S\|\left(R^{3}\right)$ (which is just

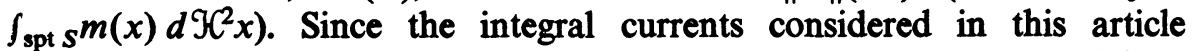
correspond to " $(\gamma, \delta)$ restricted sets" [A2], one need not be concerned here with the more pathological currents.

We will also consider top dimensional integral currents (elements of $I_{3}\left(R^{3}\right)$ ) in the case where volume constraints are used. An open set with positive orientation, finite volume, and piecewise $C^{1}$ boundary which also has finite area is represented by such a current, as is any element in the closure of the space of such sets under the distance $N$ defined by

$$
N(S, T)=\mathbf{M}(S-T)+\mathbf{M}(\partial S-\partial T) .
$$

In fact, using the strong approximation theorem [F1, 4.2.20], one can show that this closure includes all integral currents with positive orientation and multiplicity function +1 everywhere.

A very important property of integral currents is compactness: every sequence of integral currents whose supports lie in a bounded region, whose masses are bounded, and whose boundaries have bounded masses, has a subsequence which converges (in the "flat" topology-see [F1] or [F2]) to an integral current satisfying the same bounds [F2, 4.2.17].

Another notion we will use occasionally is that of a varifold. A varifold $V$, 
in the context of this article, also has a measure $\|V\|$ which is $\mathcal{H}^{2}$ restricted to a relatively nice set and a "tangent plane" function defined at $\|V\|$ almost all points; the difference is that the "tangent plane" can be independent of the actual tangent plane of the surface and it can be a probability distribution of planes, rather than a single plane. A simple example arises in \$3. If the "tangent plane" distribution does agree with the actual tangent plane to the underlying surface at $\|V\|$ almost all points, then we say $V$ is the varifold naturally associated to that surface. See [Ad] for further details.

\section{The Wulff construction and the crystal of an integrand. We call}

$$
F: G_{0}(n+1, n) \rightarrow R^{+}
$$

a (constant coefficient, codimension 1, oriented) integrand on $R^{n+1}$. Although integrands can be quite wild, to each integrand $F$ there is associated a convex oriented geometric object, its crystal (defined below), which seems to contain all the essential information about $F$. In particular, any two integrands having the same crystal also have the same geometrical solutions to the problem of minimizing-subject to having a given boundary-the integral of the integrand (Theorem 3.3). And in a sense, the wilder the integrand is, the simpler its crystal becomes, with many of the more intricate integrands having polyhedral crystals. In this case, which is the main one considered in this article, the crystal alone provides many barriers (see \$4); these are then the major tools used in proving the regularity of the solutions and in constructing some of them. There is even no need to have the integrand be bounded; extended integrands

$$
F: G_{0}(n+1, n) \rightarrow\{t: 0 \leqslant t<\infty\}
$$

have crystals, and an example of such an extended integrand is given in $\$ 2$. The basic property of the crystal of an integrand is that it is the shape having the least surface integral for the volume it contains (Theorem 2.1), and hence if $F$ is the surface tension function of a physical material, the crystal of $F$ should be the equilibrium shape of a lump of that material. (Since such problems as mass transport and nonequilibrium growth in fact occur for any sizable lump, the crystal is sometimes called the infinitesimal equilibrium shape.)

The crystal of an (extended) integrand $F$ is defined to be the result of the Wulff construction:

WulfF's CONSTRUCTION. Let $F$ be an integrand or extended integrand on $R^{n+1}$ and let $F^{*}$ be the dual function defined on the unit sphere $\partial B^{n+1}(0,1)$ by $F^{*}(v)=F\left({ }^{*} v\right)$ for each $v$ in $\partial B^{n+1}(0,1)$; here ${ }^{*} v$ denotes the dual of $v$ and hence can be regarded as an element of $G_{0}(n+1, n)$.

Plot $F^{*}$ radially: for each $v$ in $\partial B^{n+1}(0,1)$, go out a distance $F^{*}(v)$ in the direction $v$ (see Figure 1). Now at each point $F^{*}(v) v$ of this polar plot, discard the oriented half space whose boundary contains that point and has tangent plane $-\left({ }^{*} v\right)$ (see Figure 2). The result of the Wulff construction is the remaining set in $R^{n+1}$, with positive orientation, and is denoted $W_{F}$ (or $W$, if $F$ is clear from context). 


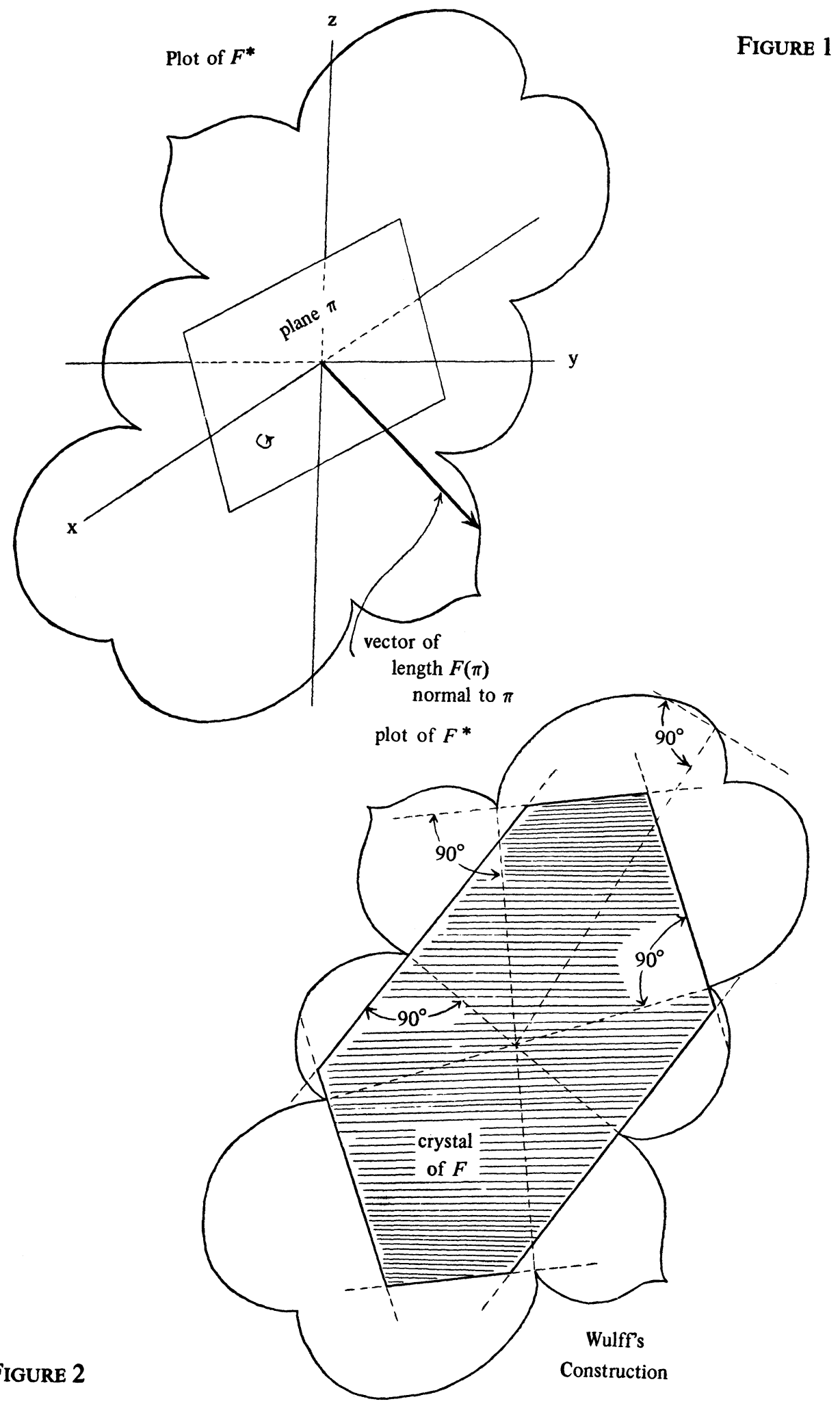


In terms of a formula,

$$
W_{F}=\bigcap_{v \in \partial B^{n+1}(0,1)}\left\{x \in R^{n+1}:\langle x, v\rangle \leqslant F^{*}(v)\right\}
$$

(with positive orientation).

An alternate description of the Wulff construction developed in [Fu] is as follows: with $\langle\cdot, \cdot\rangle$ the standard inner product on $R^{3}$ and $|\cdot|$ the standard norm, we define the operator

$$
\begin{gathered}
W: \text { integrands } \rightarrow \text { integrands, } \\
W(F)(\pi)=\inf \left\{F\left({ }^{*} w\right)\left\langle{ }^{*} \pi, w\right\rangle^{-1}: w \in R^{3},|w|=1,\left\langle{ }^{*} \pi, w\right\rangle>0\right\} \\
\text { for any integrand } F \text { and each } \pi \in G_{0}(n+1, n) .
\end{gathered}
$$

Then the crystal of $F, W_{F}$, is the open set with positive orientation whose boundary is the radial plot of the function $(W(F))^{*}$.

A property of integrands and their crystals which is obvious from the first definition of $W_{F}$ is that the crystal of any integrand is convex (and hence $\left.W_{F} \in \mathbf{I}_{n+1}\left(\mathbf{R}^{n+1}\right)\right)$. To see other properties, we follow [Fu] and define three more operators, $I, A$, and $C$, from integrands to integrands as follows: for every integrand $F$ and every $\pi \in G_{0}(n+1, n)$, we let

$$
I(F)(\pi)=1 / F(\pi)
$$

( $I$ is the multiplicative inverse operator),

$$
A(F)(\pi)=\sup \left\{F\left({ }^{*} w\right)\left\langle{ }^{*} \pi, w\right\rangle: w \in R^{3},|w|=1\right\}
$$

(so $A=I \circ W \circ I$ and $W=I \circ A \circ I$ ); we let $C(F)$ be the integrand such that the radial plot of $(C(F))^{*}$ is the boundary of the convex hull of the radial plot of $F^{*}$.

One checks that $C=W \circ A$, and for a given integrand $F$, if $H$ is any integrand which satisfies $W(H)=C(F)$, then $H \geqslant A(F)$. From this, it follows that for any convex set $K$ containing the origin in its interior, there is an integrand $F$ such that the crystal of $F$ is $K$; one such $F$ is just $A(k)$, where $k$ is the integrand such that the radial plot of $k^{*}$ is the boundary of $K$.

One property that some integrands have is convexity: an integrand $F$ is convex (equivalently, semielliptic) if and only if $C \circ I(F)=I(F)$. Note that the integrand $A(k)$ having the given convex set $K$ as its crystal is in fact a convex integrand, and hence that for any integrand $F$, there is a unique convex integrand (namely, $A \circ W(F)$ ) having the same crystal; this is also the smallest integrand having that crystal. The consequences of convexity will be discussed in the next section.

(In the terminology of [R], $(A \circ W(F))^{*}$, extended to a function on all of $R^{n+1}$ by positive homogeneity, is the support function of $W_{F}$; note that $A \circ W(F)=F$ if and only if $F$ is a convex integrand, so convex integrands correspond to the support functions of their crystals.)

Another property of the operator $A$ is less immediately obvious but quite important. A few definitions are needed. For any $h>0$, define the homothety

$$
\mu_{h}: R^{n+1} \rightarrow R^{n+1}, \quad \mu_{h}(x)=h x .
$$


For a given integrand $F$ and $h>0$, define

$$
W_{F}^{h}=\mu_{h \#}\left(W_{F}\right) \text {. }
$$

If $P \in \mathbf{I}_{n+1}\left(\mathbf{R}^{n+1}\right)$ is an open set, positively oriented, having $\partial P$ piecewise $C^{1}$ and $M(\partial P)<\infty$, and if $F$ and $h$ are given, we define $P^{h} \in \mathbf{I}_{n+1}\left(\mathbf{R}^{n+1}\right)$ by

$$
P^{h}=\left\{x+y: x \in \operatorname{spt} P, y \in \text { spt } W_{F}^{h}\right\}
$$

with positive orientation. Then for almost all $x$ such that $\overrightarrow{\partial P}(x)$ exists, we have

$$
\lim _{h \rightarrow 0} h^{-1} \operatorname{dist}\left(x, \operatorname{spt} \partial P^{h}\right)=A \circ W(F)(\overrightarrow{\partial P}(x)) .
$$

A fundamental fact about the crystal of an integrand is the following classical theorem. It is was first stated without proof by G. Wulff [W], and successively more complete proofs were given by $[\mathbf{L n}],[\mathbf{L e}],[D]$, and $[H]$, among others. A complete proof of the unoriented case in general dimensions appears in [T1] and [T2].

THEOREM 1.1 (WulfF's THEOREM). Suppose $F$ is an (extended) integrand. Then $\mathbf{F}\left(\partial W_{F}\right)<\mathbf{F}(\partial T)$ for every $T \in \mathbf{I}_{n+1}\left(\mathbf{R}^{n+1}\right)$ such that $\mathbf{M}(T)=\mathbf{M}\left(W_{F}\right), T$ is positively oriented $\|T\|$ almost everywhere, and $T$ is not $W_{F}$ or a translation of $W_{F}$.

Proof. We abbreviate $W_{F}$ by $W$. Let $P$ in $I_{n+1}\left(R^{n+1}\right)$ be an open set, positively oriented, with piecewise $C^{1}$ boundary. Then, in the terminology defined above,

$$
\begin{aligned}
\mathbf{F}(\partial P) & =\int F(\overrightarrow{\partial P}(x)) d\|\partial P\| x \\
& \geqslant \int A \circ W(F)(\overrightarrow{\partial P}(x)) d\|\partial P\| x=\lim _{h \rightarrow 0}\left(\mathbf{M}\left(P^{h}\right)-\mathbf{M}(P)\right) / h .
\end{aligned}
$$

By the Brunn-Minkowski theorem [F1, 3.2.41],

$$
\mathbf{M}\left(P^{h}\right) \geqslant\left(\mathbf{M}(P)^{1 /(n+1)}+\mathbf{M}\left(W^{h}\right)^{1 /(n+1)}\right)^{n+1} ;
$$

since $\mathbf{M}\left(W^{h}\right)=h^{n+1} \mathbf{M}(W)$ and $\mathbf{M}(W)=\mathbf{M}(P)$, we get

$$
\lim _{h \rightarrow 0}\left(\mathbf{M}\left(P^{h}\right)-\mathbf{M}(P)\right) / h \geqslant(n+1) \mathbf{M}(W) .
$$

If $P=W$, then all the inequalities above are equalities. Therefore, running back up through the argument with $W$ replacing $P$, we get $(n+1) \mathbf{M}(W)=$ $\mathbf{F}(\partial W)$, and hence $\mathbf{F}(\partial W)<\mathbf{F}(\partial P)$.

If $T$ is as in the statement of the theorem and has multiplicity $1\|T\|$ almost everywhere, then $T$ is in the $N$-closure of currents such as $P$ (see the terminology in the introduction) and if $\left\{P_{i}, i=1,2, \ldots\right\}$ is a sequence of such currents converging to some $T$ as in the theorem then $\mathbf{F}\left(P_{i}\right)$ converges to $F(T)$. The condition of multiplicity 1 can be removed since the masses of currents and their boundaries scale under $\mu_{h}$ by different powers of $h$.

Uniqueness of the solution, compared to currents $P$ with piecewise $C^{1}$ boundary as above, follows from the fact that at least one of the inequalities is strict if $P$ is not $W$ or a translation of it. Uniqueness in the larger class 
follows from [T2] and is sufficiently involved that it will not be reproduced here.

REMARK. $W$ is actually the unique solution even in a varifold sense to this problem of minimizing the surface integral subject to enclosing a given volume; see [T2].

Whether or not the crystal of $F$ is centrally symmetric (i.e. $F$ is equivalent to an integrand on the unoriented Grassmannian $G(n+1, n)$ ), the inversion of the crystal is also important; this integral current is defined by taking the central inversion of the crystal and giving it a negative orientation.

COROLlaRY. The inversion of $W_{F}$ minimizes $\mathbf{F}$ among all integral currents $T$ with $\mathbf{M}(T)=\mathbf{M}\left(W_{F}\right)$ and negative orientation.

2. Examples of Wulff construction. (1) Trivial example: $F \equiv 1$. Then the crystal of $F$ is the unit sphere. This $F$ is called the area integrand, since $F(S)$ is the $n$-dimensional area of $S$ (counting multiplicity) for any $S \in I_{n}\left(R^{n+1}\right)$. (Actually, the example is not so trivial, in that the Brunn-Minkowski inequality, which is the heart of the proof of the Wulff construction, is a standard means of proving that the sphere has least area for the volume it surrounds.)

(2) Typical example: Material with a lattice structure. Computing the energy holding a substance together by using only nearest neighbor bonds, and then obtaining a surface energy function by seeing what bonds are broken, one can arrive at an approximate surface tension function; there are also techniques for direct measurement of the surface tension function (e.g. [AC]). Figure 3 (taken from [H]) shows a typical function; the crystals of these functions are polyhedral (see [H]).

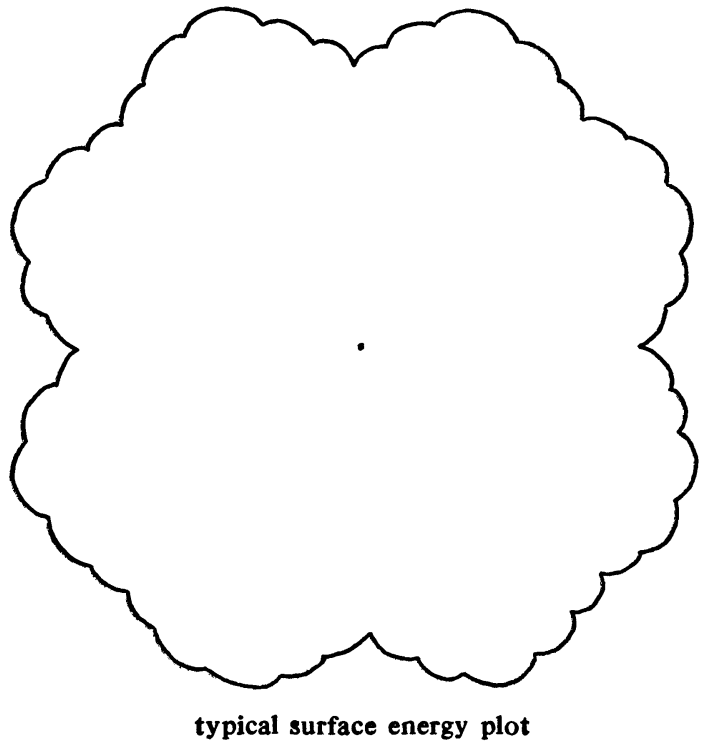

FIGURE 3

(3) Unusual example: Catching fish using a sailboat. One can use the Wulff construction to decide the best path to sail when trawling for fish with a 
sailboat. The speed a particular sailboat can sail is a function of the direction it is sailing, given a wind of constant velocity and otherwise constant conditions. The velocity profile of a typical sailboat is given in Figure 4. Let $F: G_{0}(2,1) \rightarrow R^{+} \cup\{\infty\}$ be given by $F(\pi)=$ the time to sail one mile in direction $\pi$ for each $\pi$ in $G_{0}(2,1)$ (note that $F=1 / v$ if $v$ is the velocity function). The polar plot of $F^{*}$ and the result of the Wulff construction are given in Figure 5 (note that the coordinates appear to be rotated because one plots $F^{*}$, not $F$ ). The crystal of $F$ is that vaguely fish-shaped object and is the region of given area such that the total time to sail around it is a minimum; equivalently, it is the shape of the largest area that can be surrounded in a fixed amount of time. Therefore if the fish are uniformly distributed, the most fish are surrounded in the given time by following this path. Note that the best tack and jibe angles appear automatically. The direction to sail is counterclockwise since the region surrounded is to have positive orientation; if you preferred to sail clockwise, you would use the inversion of the crystal as the best path.

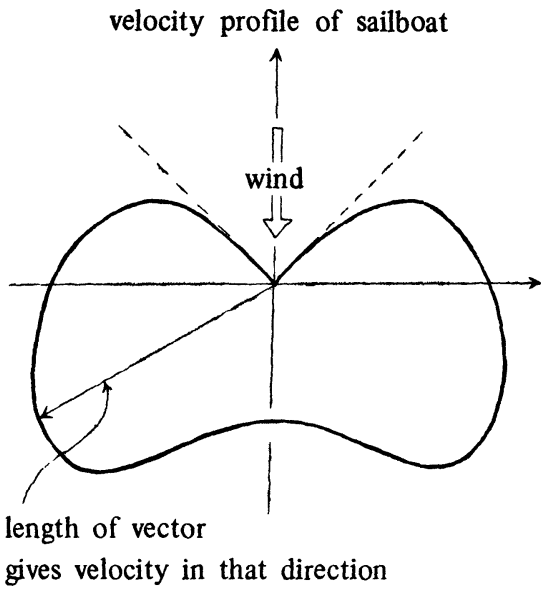

FIGURE 4

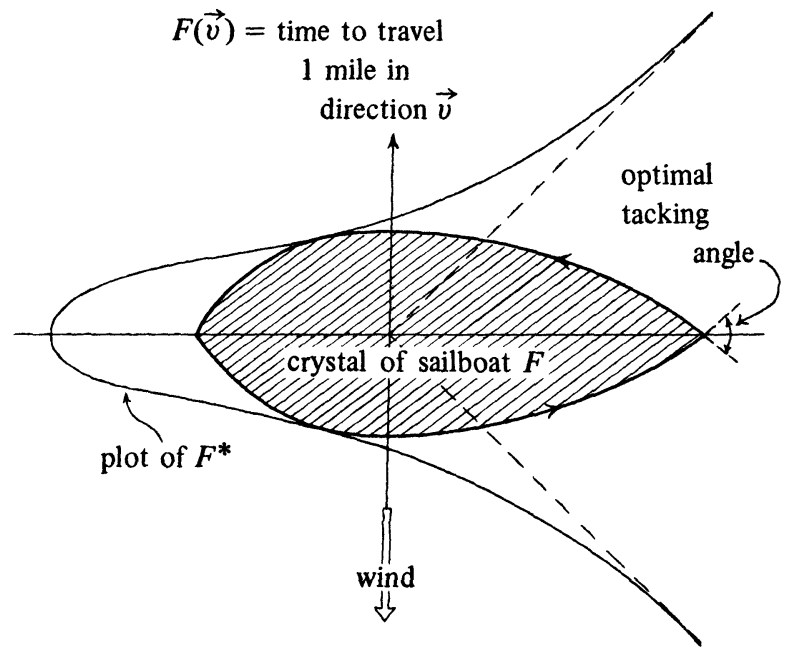

Figure 5 
3. Crystalline integrands. An integrand $F$ is defined to be crystalline if and only if its crystal is polyhedral; thus in the examples of the previous section, surface tension functions for physical crystals are crystalline, but the time function for the sailboat is not. We study crystalline integrands for several reasons: those with appropriate symmetries provide a mathematical model for questions involving surface tensions of solids; the finiteness of the number of plane directions in the boundary of the crystal leads, under certain circumstances, to a similar finiteness and computability for solutions to the prescribed boundary problem; and the interplay between elliptic integrands and crystalline integrands may yield new information on surfaces minimizing the integrals of elliptic integrands. In this section we consider that interplay and the extent to which the crystal of an integrand determines the solutions to the prescribed boundary problem for that integrand.

Crystalline integrands can clearly be either convex or not. An integrand $F$ is said to be strictly crystalline if $F$ is crystalline and

$$
F(\pi)>A \circ W(F)(\pi)
$$

whenever $\pi \in G_{0}(3,2)$ is not a tangent plane of the crystal of $F$. An illustration of a convex integrand and a strictly crystalline integrand having the same crystal is given in Figure 6. Elliptic integrands, on the other hand, are those which have uniformly convex crystals (the area integrand, for instance, is elliptic). Thus the set of elliptic integrands is disjoint from the set of crystalline integrands.

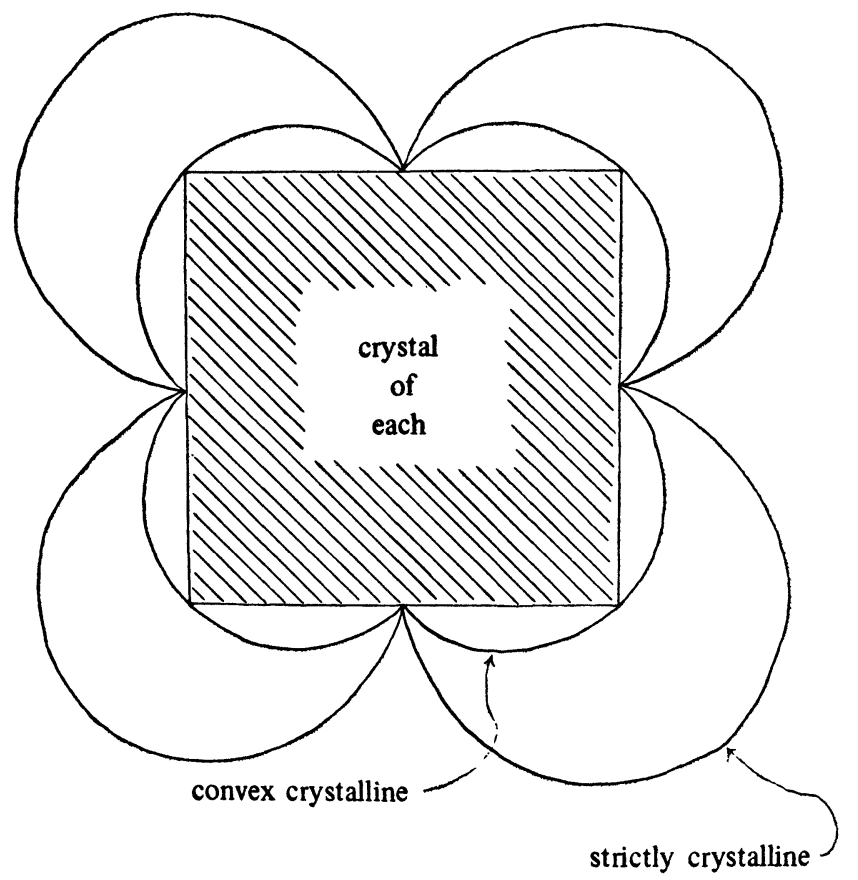

FIGURE 6

THEOREM 3.1. If $F$ is an elliptic integrand, then in the $C^{0}$ topology $F$ is in the closure of the class of crystalline integrands. If $G$ is convex and crystalline, then in the $C^{0}$ topology $G$ is in the closure of the class of elliptic integrands. 
Proof. Approximate the crystal of $F$ by convex polyhedral bodies; approximate the crystal of $G$ by uniformly convex bodies.

This theorem implies in particular that any construction procedure for surfaces minimizing the integrals of crystalline integrands will produce explicit approximations to surfaces minimizing the integrals of elliptic integrands. Since such a construction procedure seems possible (see the last section), this is another major reason for studying crystalline integrands.

Convexity of an integrand is important because it implies the lower semicontinuity of its integrals: if $S_{i}, i=1,2, \ldots$, is a sequence of integral currents converging to $S$, then $\lim \mathrm{F}\left(S_{i}\right)>\mathrm{F}(S)$. Thus we have:

Theorem 3.2. If $F$ is convex and $B \in I_{n-1}\left(R^{n+1}\right)$ with $\partial B=0$, then there exists $S$ in $I_{n}\left(R^{n+1}\right)$ with $\partial S=B$ such that $\mathrm{F}(S)<\mathrm{F}\left(S^{\prime}\right)$ for any $S^{\prime}$ in $I_{n}\left(R^{n+1}\right)$ with $\partial S^{\prime}=B$.

Proof. The direct method in the calculus of variations-taking the limit of a (sub)sequence of currents whose integrals approach the infimum-produces a solution.

One example of non-lower-semicontinuity is provided by the sailboat. Suppose you want to go directly up-wind. If you just point the boat that way, it will take infinite time, since the speed you can sail in that direction is zero (in truth, it is negative). So you make one or more tacks. Neglecting the time it takes to tack, as we did implicitly before, if you take any finite number of tacks, and always sail along one of the sides of the best tack angle in between tacks, then all paths leaving from the starting point and arriving at the finishing point require the same length of time, which is also the least possible time (Theorem 4.1). But the limit (in the usual, namely integral current, sense) of a sequence of such paths can be a path heading straight up-wind, which is clearly not a path of the same or smaller time. (See Figure 7.) (One path which does have the same time, however, is the (rotated) graph of a Cantor type function!) To make the limit also be a solution, one would have to go to varifolds, rather than integral currents; with varifolds, the limiting "tangent plane" distribution of this sequence at each point of the limit line would be half $\pi_{1}$ and half $\pi_{2}$, where $\pi_{1}$ and $\pi_{2}$ form the best tack angle.
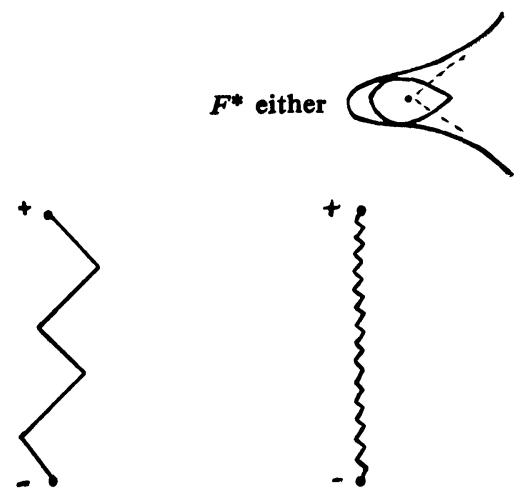

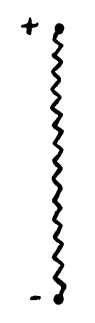

all F-minimal
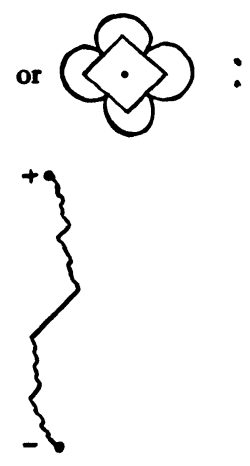

not F-minimal

FIGURE 7 
In $R^{3}$, one cannot avoid the problem by choosing other minimizing sequences. Consider the problem of taking a single crystal of a substance, such as table salt, whose surface tension function $F$ has a cube as its crystal and fixing a rectangular boundary $B$ in a plane $\pi$ parallel to one edge of the cube and at a $45^{\circ}$ angle to the other two edges (this could be accomplished, say, by painting the whole crystal with an insoluble paint and then slicing off a chunk parallel to an edge of the crystal as above), then trying to find the interface of least energy having this boundary (say by putting the painted, sliced crystal in a saturated solution of the salt so that either depositing more mass on the crystal or dissolving mass does not change the total energy of the system). This problem has no solution in the usual (integral current) sense, if $F$ is strictly crystalline: a minimizing sequence is shown in Figure 8, but there is a solution only in the varifold sense, and it is unique (Theorem 3.3 and Corollary to Theorem 4.1). Since this is a reasonable physical problem, and something must happen, our model must break down; what seems to happen in fact is that everything works fine until one reaches atomic scales, where the notion of tangent planes becomes untenable.

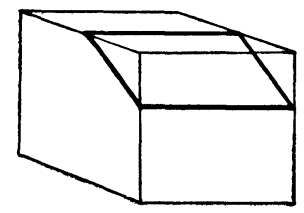

crystal and boundary

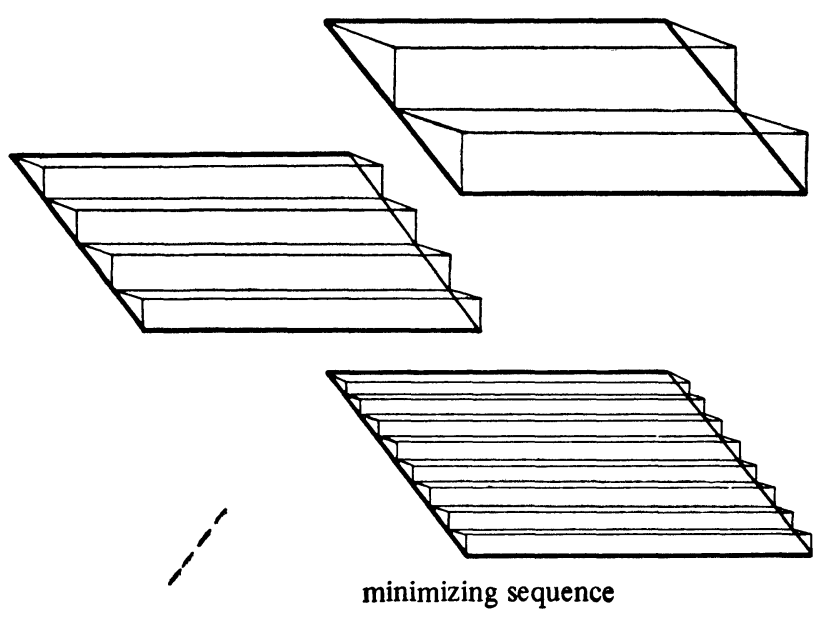

FIGURE 8

It is thus useful to consider only convex integrands if possible. It is not difficult to see that to every integrand there corresponds a unique convex integrand with the same crystal (it is the smallest integrand having that crystal); Theorem 3.3 below says that we lose nothing by using the convex integrand instead of the usual one. Before proving this theorem, we introduce several terms we need here and throughout the rest of the paper, and we prove an approximation lemma. 
Suppose $F$ is a crystalline integrand on $R^{3}$. We define the dual of the crystal as the polyhedral decomposition of the unit sphere $\partial B^{3}(0,1)$ formed by putting a vertex at every point which is a unit oriented normal to a face of the crystal and a 1-simplex (namely, the shortest path on the sphere connecting them) between every pair of vertices which correspond to adjacent plane segments in the crystal.

Given a plane $\pi$ in $G_{0}(3,2)$ and $h>0$, we define the crystal deformation to depth $h$ of $\pi$ as follows: the crystal is translated behind the plane $\pi$ ("behind" being defined with respect to the orientation of the plane) and is brought up until it penetrates the plane to a depth $h$; then the part of $\pi$ cut off by the crystal is replaced by the part of the crystal which penetrated through the plane.

Crystal deformations of an integral current $S$ around a point $x$ where spt $S$ has an oriented tangent plane $\vec{S}(x)$ are defined analogously, except that a homothety to shrink the crystal is also required so that the deformations only affect $S$ near $x$. (If $S$ has higher multiplicity at $x$, then clearly the replacement surface must be taken with the appropriate multiplicity so as to introduce no new boundary.) Note in particular that the crystal is brought up from under $S$ so that the replacement has the same boundary as the part it replaces.

For each normal $v_{i}$ to a crystal face, let $h>0$ and let $a_{i}(h)$ be the area of that face which is cut off by the crystal deformation of depth $h$, and let $a(h)$ be the area in the plane of $\pi$ which was cut out; then define $a_{i}\left(=a_{i}(\pi)\right)$ as $\lim _{h \rightarrow 0} a_{i}(h) / a(h)$. It is not hard to see that if $v$ is the unit normal of $\pi$, then $v=\Sigma a_{i} v_{i}$; this expression for $v$ is defined to be the canonical expression for $v$ and $\sum a_{i} \pi_{i}$ the canonical expression for $\pi$, where for each $i \pi_{i}$ is normal to $v_{i}$. (If only three planes meet at every corner of the crystal, then in fact the $a_{i}$ 's are uniquely defined by $v=\Sigma a_{i} v_{i}$ since one uses only vertices $v_{i}$ which are the vertices of the simplex in which $v$ lies.) By the construction of the crystal, we have $F(\pi) \geqslant \sum a_{i} F\left(\pi_{i}\right)$. (An alternative characterization of strictly crystalline is the requirement $F(\pi)>\Sigma a_{i} F\left(\pi_{i}\right)$ if $\pi$ is not parallel to a face of the crystal.)

Approximation Lemma. Suppose $F$ is crystalline and $S \in I_{2}\left(R^{3}\right)$. Then given $\varepsilon>0$ and $\eta>0$ there exist $T_{1}$ and $T_{2}$ in $I_{2}\left(R^{3}\right)$ such that $\partial S=\partial\left(T_{1}+\right.$ $\left.T_{2}\right), \mathbf{M}\left(T_{1}\right)<\eta, \vec{T}_{2}(x)$ is polyhedral with plane segments parallel to faces of $W_{F}$, and $\mathrm{F}\left(T_{2}\right)<\mathrm{F}(S)+\varepsilon$.

Proof. Apply crystal deformations to $S$ around points $x$ such that $\vec{S}(x)$ is not parallel to a face of the crystal.

THEOREM 3.3. Suppose $G$ is any crystalline integrand having the same crystal as the convex crystalline integrand $F$. Then if $S^{\prime} \in I_{2}\left(R^{3}\right)$ minimizes the integral of $G$ (among all integral currents with the same boundary), it minimizes the integral of $F$. Conversely, if $S \in I_{2}\left(R^{3}\right)$ minimizes the integral of $F$, then the varifold $V$ defined by replacing $\vec{S}(x)$ (wherever it is defined) by its canonical expression minimizes the integral of $G$ in the family of all varifolds which are varifold limits of integral currents with boundary $\partial S ; V$ minimizes $\mathbf{F}$ uniquely in this family if $F$ is strictly crystalline and $S$ uniquely minimizes $G$.

Proof. Suppose $S^{\prime}$ minimizes the integral of $G$, and there exists $S$ such that 
$\mathbf{F}(S)<\mathbf{F}\left(S^{\prime}\right)$ and $\partial S=\partial S^{\prime}$. Applying the approximation lemma above to $S$, with $\varepsilon=\left(F\left(S^{\prime}-F(S)\right)\right) / 2$ and $\eta=\varepsilon / \max _{G_{0}(3,2)} G$, we obtain $T_{1}$ and $T_{2}$ such that $\partial\left(T_{1}+T_{2}\right)=\partial S=\partial S^{\prime}$ and

$\mathbf{G}\left(T_{1}+T_{2}\right) \leqslant \varepsilon / 2+\mathbf{G}\left(T_{2}\right)=\varepsilon / 2+\mathbf{F}\left(T_{2}\right) \leqslant \varepsilon+\mathbf{F}(S)<\mathbf{F}\left(S^{\prime}\right) \leqslant \mathbf{G}\left(S^{\prime}\right)$,

a contradiction of the fact that $S^{\prime}$ minimizes $\mathbf{G}$.

Conversely, suppose there exists a varifold $V^{\prime}$ in the family described in the theorem, which satisfies $\mathbf{G}\left(V^{\prime}\right)<\mathbf{G}(V)$. Let $S^{\prime}$ be the current limit of some sequence of currents giving $V^{\prime}$. Then $\mathbf{G}\left(V^{\prime}\right)>\mathbf{F}\left(V^{\prime}\right)>\mathbf{F}\left(S^{\prime}\right)$ and $\mathbf{G}(V)=$ $\mathbf{F}(V)=\mathbf{F}(S)$. Thus $\mathbf{G}\left(V^{\prime}\right)<\mathbf{G}(V)$ implies $\mathbf{F}\left(S^{\prime}\right)<\mathbf{F}(S)$, contradicting the F-minimality of $S$. Uniqueness follows from the definition (or alternate characterization) of strictly crystalline.

4. Some basic F-minimal surfaces. The most basic surfaces used in studying crystalline problems are those of the following theorem, primarily because of their usefulness as barriers in the following sense.

Suppose one knows that a given surface $S$ is uniquely F-minimal for its boundary $B$. Then if $S^{\prime}$ is another $F$-minimal surface (with boundary $B^{\prime}$ ), and if (spt $B \cap$ spt $\left.S^{\prime}\right) \cup\left(\right.$ spt $B^{\prime} \cap$ spt $S$ ) $=\varnothing, S^{\prime}$ cannot cross $S$, as every subset of $S$ is also uniquely $F$-minimal for its boundary. Thus a uniquely $F$-minimal surface is a barrier for other F-minimal surfaces. Crystalline integrands are particularly rich in simple barriers-one large class of such uniquely F-minimal surfaces is isolated below, and the other surfaces of the theorem become barriers also when the volume-maximizing condition of the next section is invoked.

THEOREM 4.1. The oriented tangent cones to the boundary of the crystal and to its inversion are $\mathbf{F}$-minimal. If $\mathbf{F}$ is crystalline, such tangent cones which have only one or two plane segments are uniquely F-minimal.

Proof. To show that a tangent plane to the crystal is uniquely F-minimal it suffices to observe that there exists a uniformly convex integrand $G$ such that $G \leqslant F$ on all of $G_{0}(3,2)$ and $G=F$ precisely on the given crystal tangent plane direction. Then if $S$ is the tangent plane, restricted to the unit disk, and $S^{\prime}$ is any other integral current with the same boundary as $S, \mathbf{F}\left(S^{\prime}\right) \geqslant \mathbf{G}\left(S^{\prime}\right)$ $>\mathbf{G}(S)=\mathbf{F}(S)$.

In the general case, we use the fact that the crystal has minimum surface integral for the volume enclosed. Consider first the case where $F$ is crystalline. Let $p$ be in spt $\partial W_{F}$ and again let $S$ be the tangent cone at $p$, restricted to the unit ball. Let $S^{\prime}$ be any other current with the same boundary and suppose $\mathbf{F}(S)-\mathbf{F}\left(S^{\prime}\right)=c>0$. If the 3-dimensional current $T$ in $R^{3}$ bounded by $S^{\prime}-S$ were such that $\int^{*} \vec{T} d\|T\|$ were nonnegative then we would have an immediate contradiction of Wulff's theorem, since if we replaced part of the crystal boundary in a neighborhood of $p$ by an appropriate homothetic reduction and translation of $S^{\prime}$, we could obtain a surface surrounding the same or greater volume as the crystal and yet having a smaller surface integral. If the volume of $T$ were negative, we could take a sequence of positive numbers $r_{i}, i=1,2, \ldots$, converging to zero, and look at the sequence of currents $C\left(r_{i}\right)$ obtained by (1) replacing the boundary of the crystal in the $r_{i}$-neighborhood of $p$ by the translation to $p$ of $\mu_{r_{i} \#} S^{\prime}\left(\mu_{r}\right.$ is the 
homothety as defined in the Wulff construction) and then (2) expanding the current bounded by this new boundary by the homothety $\mu_{s}$, where $s=(1-$ $\left.v r_{i}^{3}\right)^{-1 / 3}$. One obtains a current $C\left(r_{i}\right)$ of the original mass. We observe that

$$
\mathbf{F}\left(C\left(r_{i}\right)\right)=s^{2}\left(\mathbf{F}\left(W_{F}\right)-c r_{i}^{2}\right)=\left(\mathbf{F}\left(W_{F}\right)-c r_{i}^{2}\right)\left(1+2 v r_{i}^{3} / 3+O\left(r_{i}^{3}\right)\right) .
$$

Thus $\mathbf{F}\left(C\left(r_{i}\right)\right)-\mathbf{F}\left(W_{F}\right)$ must become positive for some $i$, a contradiction of the fact that the crystal is F-minimal for the volume it contains.

If $F$ is not crystalline, then the comparison surface $S^{\prime}$ as above must be patched into the crystal boundary. However, with a small enough radius $r$ in the homothety one can make the extra integral small in comparison to $\left(\mathbf{F}(S)-\mathbf{F}\left(S^{\prime}\right)\right) r^{2}$ and make the volume change also small; then the above argument gives the first part of the theorem.

The same proof, with negative and positive interchanged throughout, shows that tangent cones to the inversion of the crystal are minimal.

The proof of the final uniqueness part of the theorem is not difficult but is somewhat long. Let the tangent cone under consideration be composed of half planes with duals $v_{1}$ and $v_{2}$. Let $v_{3}$ and $v_{4}$ be the duals to the "end planes" of the corresponding edge on the crystal (see Figure 9(a)). (If there are, say, $k>1$ planes instead at, say, the $v_{3}$ end, then $v_{3}$ must be replaced by $b_{3,1} v_{3,1}+\cdots+b_{3, k} v_{3, k}$ in what follows for appropriate $\left\{b_{3, i}\right\}$.)
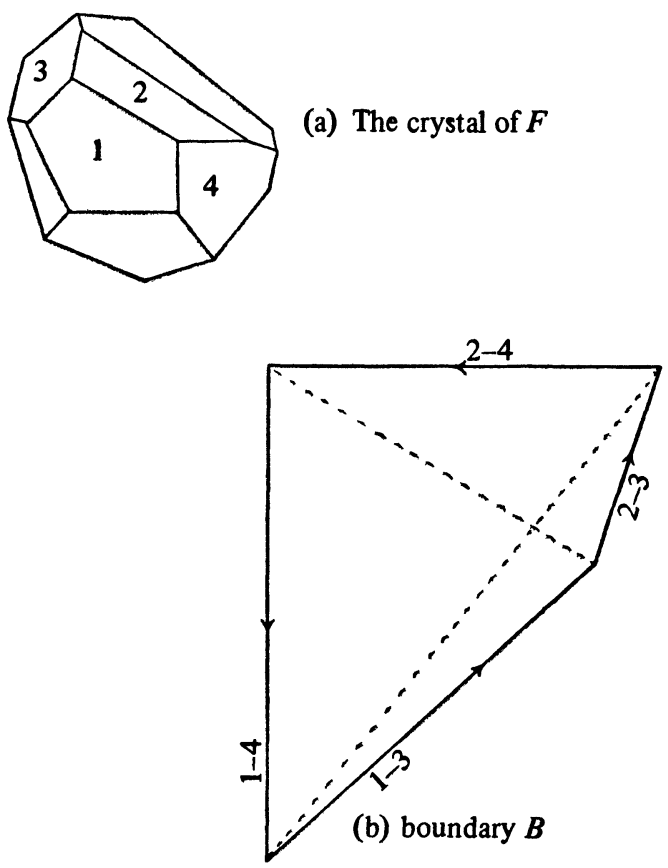

FIGURE 9

Fix a boundary $B$ as in Figure 9(b); it is sufficient to show that the tangent cone, restricted to the convex hull of this boundary, is uniquely F-minimal. This boundary was chosen so that it is spanned not only by oriented triangles with normals $v_{1}$ and $v_{2}$ but also by oriented triangles with normals $v_{3}$ and $v_{4}$. Let the areas of these triangles be $b_{1}, b_{2}, b_{3}$, and 1 respectively. Since the first 
pair of triangles minus the second pair form a cycle, $b_{1} v_{1}+b_{2} v_{2}=b_{3} v_{3}+v_{4}$, but since the plane segments with normals $v_{3}$ and $v_{4}$ do not meet in the crystal,

$$
b_{1} F^{*}\left(v_{1}\right)+b_{2} F^{*}\left(v_{2}\right)=b_{3} F^{*}\left(v_{3}\right)+F^{*}\left(v_{4}\right)-A
$$

for some $A>0$.

If $S$ is any polyhedral surface having this boundary, let its plane segments have normals $v_{1}, \ldots, v_{n}$ and corresponding areas $a_{1}, \ldots, a_{n}$. For each $i$, write $v_{i}=d_{1} v_{1}+d_{2} v_{2}+d_{4} v_{4}$. If $d_{4} \geqslant 0$, set $c_{i j}=d_{j}$ for $j=1,2,4$ and $c_{i, 3}=0$; otherwise, set $c_{i, 1}=d_{1}+d_{4} b_{1}, c_{i, 2}=d_{2}+d_{4} b_{2}, c_{i, 3}=-d_{4} b_{3}$, and $c_{i, 4}=0$ and observe that we still have $v_{i}=\sum_{j} c_{i, j} v_{j}$. Let $c_{j}^{\prime}=\sum_{i} a_{i} c_{i, j}$ for $j=1, \ldots, 4$ and note that $c_{4}^{\prime} \geqslant \max \left\{c_{4, i}\right\}>0$ unless $S$ itself is the restricted tangent cone. Since the restricted tangent cone minus $S$ is a cycle again, we have

$$
0=b_{1} v_{1}+b_{2} v_{2}-\sum_{i=1}^{n} a_{i} v_{i}=b_{1} v_{1}+b_{2} v_{2}-\sum_{j=1}^{4} c_{j}^{\prime} v_{j}
$$

so that $b_{1}-c_{1}^{\prime}=c_{4}^{\prime} b_{1}, b_{2}-c_{2}^{\prime}=c_{4}^{\prime} b_{2}$, and $c_{3}^{\prime}=c_{4}^{\prime} b_{3}$.

Because all tangent cones to the crystal are F-minimal,

$$
F^{*}\left(v_{i}\right) \geqslant \sum_{j=1}^{4} c_{i j} F^{*}\left(v_{j}\right)
$$

for each $i=1, \ldots, n$ (to see this, rearrange so that all coefficients on each side are nonnegative), and thus

$$
\begin{aligned}
\mathbf{F}(S) & =\sum_{i=1}^{n} a_{i} F^{*}\left(v_{i}\right) \\
& >\sum_{j=1}^{4} c_{j}^{\prime} F^{*}\left(v_{j}\right)=b_{1} F^{*}\left(v_{1}\right)+b_{2} F^{*}\left(v_{2}\right)+A c_{4}^{\prime} .
\end{aligned}
$$

This shows that among polyhedral surfaces, the restricted tangent cone is uniquely F-minimal. Assume there exists an integral current $S$ with $\partial S=B$ and $F(S)=b_{1} F^{*}\left(v_{1}\right)+b_{2} F^{*}\left(v_{2}\right)$; we first note that the proof of minimality shows that none of $S$ can lie above the tangent cone. Then we deform $S$ by a crystal deformation involving the corner whose planes have normals $v_{1}, v_{2}$, and $v_{4}$ and note that $S$ not equal to the tangent cone implies that for some such deformation (which doesn't move the boundary), there is an open region of positive area $a$ on the $v_{4}$ plane; since this cone is F-minimal, the deformation cannot have increased the integral. We now take a sequence of polyhedral approximations to this deformed $S$ as in the Approximation Lemma of $\$ 3$, and which each include a segment having area at least $3 a / 4$ and normal $v_{4}$. By the above, for small enough $\varepsilon$, each polyhedral approximation has extra integral (compared to the tangent cone) of at least $A a / 2$, and hence for small enough $\eta, S$ has extra integral at least $A a / 4$, which contradicts the assumption that $S$ is F-minimal.

5. The volume maximizing condition. Situations like that in the example of 83, where the graphs of even Cantor-type functions can be F-minimal for a 
given boundary, illustrate that surfaces minimizing the integrals of nonelliptic integrands can be quite irregular without some further selection. The situation becomes considerably simplified if, in the class of all integral currents minimizing the integral of $F$, subject to having a given boundary, one looks only at those satisfying a local volume maximizing principle. One such condition is that of obeying local crystal corner barriers:

If at some point $x$ in spt $S$ there exists a neighborhood $N$ of $x$ such that a tangent cone to a corner of the crystal can be pushed up through $S$ restricted to $N$ as in a crystal deformation without moving $\operatorname{Bndry}(N \cap \operatorname{spt} S)$ then $S$ does not obey local crystal corner barriers. If for every point $x$ in $S$ there is no such neighborhood, then $S$ does obey local crystal corner barriers.

THEOREM 5.1. Let $B$ in $I_{1}\left(R^{3}\right)$ have $\partial B=0$ and let $F$ be a convex crystalline integrand. Then there exists $S$ in $I_{2}\left(R^{3}\right)$ such that $\partial S=B, S$ is F-minimal among all integral currents with boundary $B$, and $S$ obeys local crystal corner barriers. Furthermore, such an $S$ has all its tangent planes normal to the 1-skeleton of the dual of the crystal.

Proof. Existence of such a locally volume maximizing solution follows from the compactness of the set of surfaces which minimize $F$ and the $F$-minimality of tangent cones to corners of the crystal of $F$. If for some $x$, $\vec{S}(x)$ is not normal to some element of the dual, then the canonical expression for $\vec{S}(x)$ produces a crystal corner whose tangent cone can be pushed up through $S$ in a small neighborhood of $x$.

COROLlaRY. All tangent cones to the crystal of $F$ are barriers for $F$-minimal surfaces which obey local crystal corner barriers.

Imposition of this volume maximizing criterion does not really decrease the amount of information available about general solutions: once one has volume maximizing solutions, pushing the corners back in again will give all the other solutions. In fact, one could just as easily go to the opposite extreme and ask for "volume minimizing" solutions, using the inversion of the crystal rather than the crystal. Either condition would give the finiteness of the next section. And this selection of a volume maximizing solution seems to make sense in the physical model that crystalline integrands are designed to describe, at least whenever the interface under consideration is between two different materials (or two different lattice orientations), as opposed to a lattice translation [C].

6. Finiteness. One can reduce the set of possible tangent planes of an F-minimal surface to a finite set by putting conditions on the support of the boundary. The conclusions of the following theorem are not nearly the best possible, but their proof indicates with a minimum of effort the basic reason for that finiteness. Note that the set of boundaries satisfying the theorem is $C^{0}$-dense in the space of all boundaries.

THEOREM 6.1. Suppose $F$ is convex and crystalline. If $B \in I_{1}\left(R^{3}\right)$ has $\partial B=0$ 
and spt $B$ piecewise linear, with each linear segment parallel to an edge of the crystal, and if $S \in I_{2}\left(R^{3}\right)$ has $\partial S=B$, is (locally) F-minimal, and obeys local crystal corner barriers, then $\|S\|$ almost all points in spt $S$ have a tangent plane in the finite set $P$ consisting of those planes which are spanned by lines parallel to edges of the crystal.

Proof. Suppose $F, S$ and $P$ are as in the statement of the theorem, and at $p \in \operatorname{spt} S, \vec{S}(p)$ is not in $P$. Then $\vec{S}(p)$ is normal to the 1-skeleton of the dual of the crystal and thus has canonical expression $a_{1} \pi_{1}+a_{2} \pi_{2}$ for some planes $\pi_{1}$ and $\pi_{2}$ which are parallel to adjacent faces of the crystal. Without loss of generality we may assume that $\vec{S}(p)=R^{2} \times\{0\}$, with its usual orientation, that $p=0$, and that the common line of $\pi_{1}$ and $\pi_{2}$ (which is of course parallel to an edge of the crystal) is $R^{1} \times\{(0,0)\}$. Since $\vec{S}(p)$ is not in $P$, no boundary segment can lie in $R^{1} \times[-\varepsilon, \varepsilon] \times\{0\}$ for some $\varepsilon>0$. We may use the fact that we only ask for almost all points in the theorem to assume $p$ and $\varepsilon$ are such that if $N=R^{1} \times[-\varepsilon, \varepsilon] \times\left[-\varepsilon^{2}, \varepsilon^{2}\right]$, then $N \cap$ spt $B$ is empty; we may also assume that $\varepsilon$ is less than half the altitude of the triangle with base 1 and sides $a_{1}$ and $a_{2}$. If spt $S \cap N$ has more than one connected component, we consider only the connected component of the origin in what follows. The boundary of spt $S \cap N$, except for the parts in $R^{1} \times\{-\varepsilon, \varepsilon\} \times$ $R^{1}$, now is seen to lie on the planes parallel to the $\left(x_{1}, x_{2}\right)$ plane and at a distance $\varepsilon^{2}$ above it and below it. Suppose it is entirely above the $\left(x_{1}, x_{2}\right)$ plane; then the tangent cone to the crystal which involves just the planes $\pi_{1}$ and $\pi_{2}$ can be pushed up through $S$ near $p$ without moving spt $S \cap$ Bndry $N$ or spt $B$; since this tangent cone is uniquely $F$-minimal, $S$ could not have been F-minimal, a contradiction. Similarly, if this part of the boundary of spt $S \cap N$ lay entirely below the $\left(x_{1}, x_{2}\right)$ plane, the tangent cone to the inversion of the crystal involving just the planes $\pi_{1}$ and $\pi_{2}$ would provide a contradiction. Finally, we observe that for $x_{1}>0$, the part of the boundary not off to the sides must be either entirely above the $\left(x_{1}, x_{2}\right)$ plane or entirely below it, since there is no boundary between $-\varepsilon^{2}$ and $+\varepsilon^{2}$; the same is true for $x_{1}<0$. Therefore from one end or the other a tangent cone to a corner of the crystal can be pushed up under $S$ without moving spt $S \cap$ Bndry $N$ or $B$, contradicting the volume-maximizing property of $S$. Thus we conclude that the set of points $p$ where $\vec{S}(p)$ is not in $P$ must have two-dimensional measure 0.

One would hope that this finiteness would imply that some finite explicit procedure for the computation of $F$-minimal surfaces under these conditions would be possible. This is in fact the case and such a procedure is outlined in the next section. Before we leave this section, however, let us investigate the conditions under which the set $\boldsymbol{P}$ of planes in the theorem above is just the set of directions of the faces of the crystal itself, and hence under which the convexity condition on $F$ can be removed without having to go to varifold solutions.

Proposition 6.2. Suppose that $G$ is crystalline and $F$ is the convex integrand with the same crystal as $F$. Suppose that every face of this crystal has an even number of edges and that for every face, opposite edges are always parallel. Then: 
(1) The plane determined by lines through the origin parallel to any two edges of the crystal is parallel to two faces of the crystal (of opposite orientation).

(2) Under the conditions of the preceding theorem, $S$ is in fact $\mathbf{G}$-minimal.

Proof. (1) Given a line through the origin parallel to an edge of the crystal, the unit oriented normals to the faces which contain that edge all lie in the great circle whose plane is perpendicular to that line. So for the given two, their corresponding great circles intersect twice. The points of intersection must also be normals to faces of the crystal; if they were not, the closest normals to each side of an intersection point would represent four faces of the crystal, opposite pairs of which have to intersect along edges parallel to those given lines, and the crystal being convex prohibits this.

(2) By the previous theorem, the set of possible tangent planes must be (except at a set of measure zero) those spanned by edges of the crystal, which by (1) above are simply the planes parallel to faces of the crystal. By Theorem 3.3, if $S$ is F-minimal, then the varifold based on $S$ is G-minimal; but this varifold has its tangent plane distribution agreeing with the tangent planes of $S$ itself and hence $S$ is G-minimal.

7. Computation of $F$-minimal surfaces. The finite number of possible tangent planes gives hope that F-minimal surfaces which locally maximize volume may be computable under appropriate boundary conditions. An explicit finite procedure for producing at least one integral current which at least locally minimizes $\mathbf{F}$ now exists if the support of the boundary $B$ lies on the boundary of a convex set (and is piecewise linear with each piece being parallel to an edge of the crystal).

This procedure has three stages, which can be summarized as follows:

(1) Cut in, with cones derived from the crystal, at the part of the boundary of the negatively oriented convex set which has boundary $B$. A surface which obeys crystal corner barriers is produced.

(2) Remove nonminimal intersections of planes by inserting segments of the crystal appropriately, and (if necessary) separate some plane segments into two or more segments-with appropriate segments of the crystal as patches between them-according to a fairly simple criterion.

(3) Vary the position of each plane segment whose position is not fixed from both sides by local crystal barriers (this includes varying planes off the boundary line segments, with appropriate patching from the crystal) to find the best overall position. (This amounts to solving a particular system of linear equalities and inequalities.)

The resulting surface satisfies five local properties which can then be shown to imply that the surface is locally F-minimal. A complete description of the procedure and the proof that it produces a surface which is locally F-minimal will be published later.

Another F-minimal surface can be obtained by working with the other half of the boundary of the convex set; it will produce a "locally volume minimizing" surface of the wrong orientation which can be converted to a locally volume maximizing surface of the right orientation simply by changing orientation and then applying local corner barriers. If this second surface agrees with the surface obtained by the first procedure, then in fact all 
F-minimal surfaces can be determined from it.

An undergraduate student at Rutgers University is currently attempting to put this procedure on a computer. Some examples of F-minimal surfaces obtained by the procedure for various $F$ 's are shown in Figures 10 and 11.

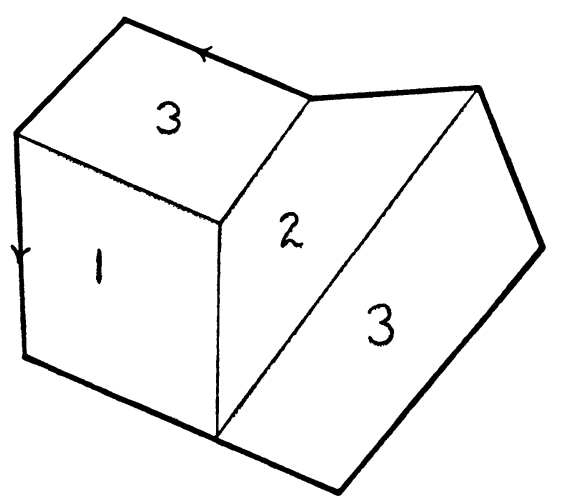

F-minimal surfaces

"maximizing volume"

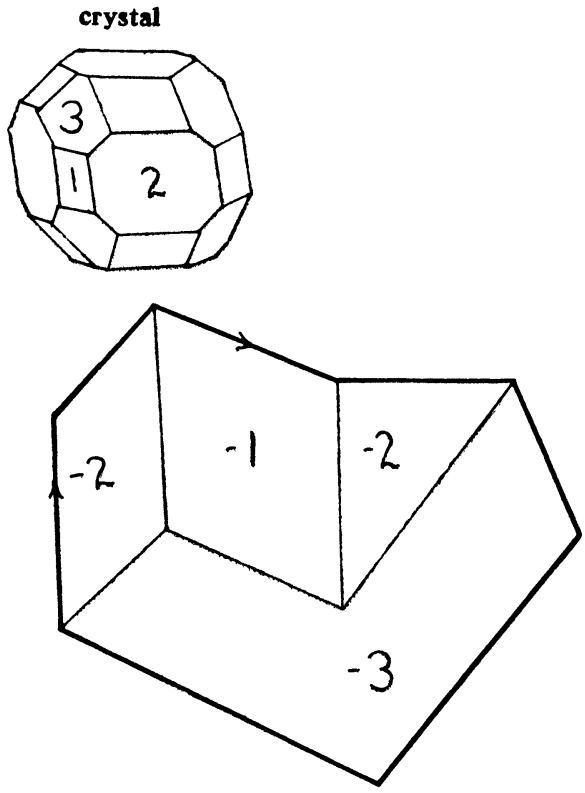

FIGURE 10

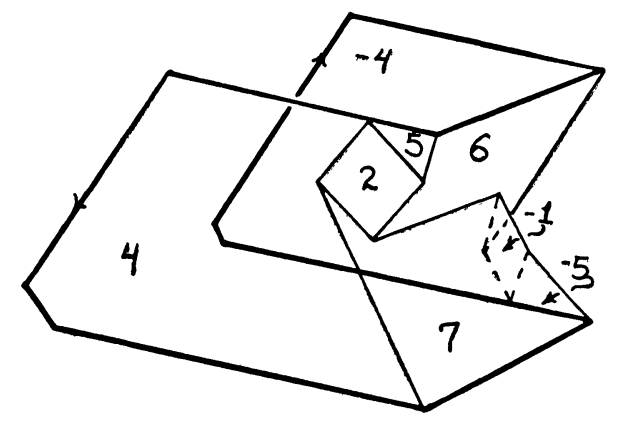

uniquely F-minimal surfaces

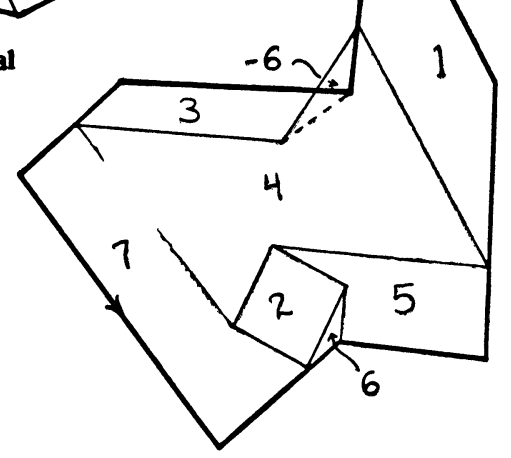

FIGURE 11

Several comments should be made about the significance of this procedure. First of all, it explicitly produces exact solutions to a large class of problems 
which are reasonable mathematical models for physical phenomena involving surface tension. (Note that the finiteness of the number of plane segments means that atomic dimensions can easily be avoided, making surface tension a meaningful concept.) Secondly, since any integrand in $R^{3}$ (in particular, any elliptic integrand) can be approximated by crystalline integrands (Theorem 3.1), the procedure produces a sequence of explicit approximations to surfaces minimizing the integral of the original integrand. (Approximations for area minimizing surfaces with boundaries on convex sets when there is known to be a unique solution have also been found by $\mathrm{H}$. Parks, but by an entirely different method [P].) Thus, in particular, we now know that extreme locally area-minimizing surfaces can be approximated by surfaces obtained in this rather straightforward three-stage procedure.

A major objective is to extend the procedure (and its proof) to higher dimensions (most of the material of this article except for the construction fairly easily extends already). It is hoped that the construction, together with the approximating procedure, will give new information on the structure as well as the accessibility of surfaces minimizing any codimension one integrand.

\section{REFERENCES}

[Ad] W. K. Allard, On the first variation of a varifold, Ann. of Math. 95 (1972), 417-491.

[A1] F. J. Almgren, Jr., Existence and regularity almost everywhere of solutions to elliptic variational problems among surfaces of varying topological type and singularity structure, Ann. of Math. 87 (1968), 321-391.

[A2] _ Existence and regularity almost everywhere of solutions to elliptic variational problems with constraints, Mem. Amer. Math. Soc. No. 165, 1976.

[AC] E. Arbel and J. W. Cahn, A method for the absolute measurement of anisotropic solid-melt surface free energies (preprint).

[C] J. W. Cahn, personal communication.

[D] A. Dinghas, Über einen geometrischen Satz von Wulff für die Gleichgewichtsform von Kristallen, Zeitschrift für Kristallographie 105 (1944), 304-314.

[F1] H. Federer, Geometric measure theory, Springer-Verlag, New York, 1969.

[F2] _ Colloquium lectures on geometric measure theory, Summer Meeting of the Amer. Math. Soc. (Seattle, Washington, August 15-18, 1977), Bull. Amer. Math. Soc. 84 (1978), 291-338.

[Fu] J. H. G. Fu, A mathematical model for crystal growth and related problems, Junior paper, Princeton University.

[H] C. Herring, Some theorems on the free energy of crystal surface, Phys. Rev. 82 (1951), 87-93.

[Le] M. v. Laue, Der Wulfssche Satz für die Gleichgewichtsform von Kristallen, Zeitschrift für Kristallographie 105 (1943), 124-133.

[Ln] H. Liebmann, Der Curie-Wulff'sche Satz über Combinationsformen von Krystallen, Zeitschrift für Krystallographie und Mineralogie 53 (1914), 171-177.

[P] H. R. Parks, Explicit determination of area minimizing hypersurfaces, Duke Math. J. 44 (1977), 519-534.

[R] R. T. Rockafellar, Convex analysis, Princeton Univ. Press, Princeton, N. J., 1970.

[T1] J. E. Taylor, Existence and structure of solutions to a class of nonelliptic variational problems, Symposia Mathematica 14 (1974), 499-508.

[T2] Unique structure of solutions to a class of nonelliptic variational problems, Proc. Sympos. Pure Math., vol. 27, Amer. Math. Soc., Providence, R.I., 1974, pp. 481-489.

[W] G. Wulff, Zur Frage der Geschwindigkeit des Wachsthums und der Auflösung der Krystallflachen, Zeitschrift für Krystallographie und Mineralogie 34 (1901), 449-530.

Department of Mathematics, Rutgers University, New Brunswick, New Jersey 08903 School of Mathematics, Institute for Advanced Study, Princeton, New Jersey 08540 